\title{
OBSERVATIONS SUR LA REPRODUCTION ARTIFICIELLE DE L'OMBRE COMMUN (THYMALLUS THYMALLUS)
}

\author{
H. CARMIE *, B. MORELET **, G. MAISSE **, L. JONARD *, R. CUINAT *
}

* Délégation Régionale du Conseil Supérieur de la Pêche, 84, avenue du Puy-de-Dôme, 6300 CLERMONT FERRAND, France.

* I.N.R.A., Laboratoire de Physiologie des Poissons, Campus de Beaulieu, 35042 RENNES Cedex, France.

\section{RESUME}

Diverses observations et résultats d'expériences réalisées à la Salmoniculture du Conseil Supérieur de la Pêche à Augerolles sont présentés, concernant les techniques de reproduction artificielle adaptées à la physiologie de l'Ombre commun. Les caractéristiques physiologiques mises en évidence sont proches de celles de la Truite avec cependant une sensibilité accrue aux basses températures, qui peuvent ralentir très fortement la fin de la maturation ovocytaire. Ces observations et expériences conduisent les auteurs à formuler un certain nombre de conseils pratiques

\section{SUMMARY}

\section{OBSERVATIONS ON ARTIFICIAL SPAWNING OF GRAYLING (THYMALLUS THYMALLUS)}

Various observations and results of experiments carried out in the hatchery of the "Conseil Supérieur de la Pêche" in Augerolles are presented, concerning the technics of artificial spawning adapted to the physiology of grayling. It is shown that the physiological characteristics are nearly the same as those of brown trout. Nevertheless high sensitivity to low temperatures reduces the speed of the final oocyte maturation. These observations and experiments lead the authors to give practical recommendations.

\section{I - INTRODUCTION}

L'Ombre commun (Thymallus thymallus L.)est très recherché par les pêcheurs, principalement par ceux opérant à la mouche artificielle. Malheureusement, seules quelques rivières abritent encore des populations naturelles : hauts bassins de l'Allier, de la Loire, du Rhin, du Rhône, de la Saône et de la Vienne (PERSAT, 1976).

La raréfaction de l'Ombre commun est en partie liée à la dégradation de son biotope. Les secteurs de cours d'eau classés dans la zone à Ombre, caractérisée par HUET (1949), cnt été fortement perturbés ces dernières décennies par la détérioration de la qualité de l'eau, l'extraction de granulats. et la construction de complexes hydro-électriques.

La pêche a probablement aussi joué un rôle appréciable dans cette raréfaction, d'autant plus que celle-ci s'exerçait souvent sur des sujets immatures (l'O mbre a une croissance rapide).

En contre-partie les ensemencements s'intensifient chaque année, d'une part dans les rivières où l'O mbre est absent bien que les caractéristiques morphodynamiques et la qualité de l'eau soient a priori favorables, d'autre part dans les cours d'eau ou une partie de son cycle est enrayée : impossibilité d'accéder aux zones de frai, colmatage des gravières...

Les sujets déversés sont généralement issus d'œufs importés de l'étranger : Pays scandinaves, Allemagne, Yougoslavie. Les produits sexuels sont prélevés sur des géniteurs capturés en milieu naturel quelques semaines avant la reproduction (SVETINA, 1958) ou en période de frai (PHILIPPART et D'HULSTERE, 1976 : STEIN et WINTERSPERGER, 1980 ; BILLARD, 1981 ; D'HULSTERE et PHILIP. PART, 1982). Ces mêmes auteurs soulignent l'impossibilité ou la grande difficulté de maîtriser en pisciculture certaines phases du cycle de l'Ombre

La Délégation Régionale "Auvergne-Limousin" du Conseil Supérieur de la Pêche a procédé en 1973 à des essais d'élevage à la Salmoniculture expérimentale d'Augerolles (Puy-de-Dôme). Un cheptel de géniteurs entièrement élevés en pisciculture a ainsi été constitué, pour la première fois semble-t-il en Europe, et permet un approvisionnement régulier en ovules tout en palliant aux aléas de la pêche de géniteurs sauvages : conditions hydrauliques défavorables, stress des poissons suivi du blocage de l'ovulation ou de mortalités, diminution du potentiel de reproduction naturelle...

Ce document fait le point sur les connaissances acquises ces dernières années sur la reproduction de l'Ombre commun en élevage, et plus particulièrement sur les observations et les résultats obtenus lors des deux dernières campagnes de pontes (1983 et 1984). 


\section{2 - MATÉRIEL ET MÉTHODES}

Toutes les expérimentations ont été faites à la Salmoniculture du C.S.P. à Augerolles. Cet établissement a été décrit par CUINAT, 1978. Depuis cette date, de nouveaux bassins ont été mis en place, notamment des bacs de $2 \times 2 \mathrm{~m}$ de type suédois.

\subsection{Le cheptel de géniteurs}

\subsubsection{Origine}

Des alevins issus de la reproduction artificielle de géniteurs capturés sur l'Allagnon (HauteLoire) en 1973, et d'œufs de souches scandinave et bavaroise ont été mis en élevage à la Salmonicul. ture d'Augerolles. Par sélection massale un lot de reproducteurs de première génération en pisciculture a été constitué.

En 1976 des croisements non contrôlés ont permis l'obtention de sujets de deuxième génération en pisciculture (lot no 1), réformés après les pontes de 1983 ou 1984. Parmi leur descendance nous avons conservé des individus qui se sont reproduits pour la première fois en 1982, à l'âge de 3 ans (lot n०2). C'est sur cette cohorte qu'ont porté les observations et les expériences réalisées en 1983 et 1984.

\subsubsection{Caractéristiques morphologiques avant la ponte}

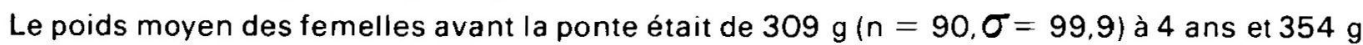
( $n=68, \sigma=95,2$ ) à 5 ans.

Les valeurs du coefficient de condition moyen des femelles à 4 ans $(1,21$, longueur à la fourche) et à 5 ans $(0,98$, longueur totale) indiquaient un bon état général de ces individus (Salmoniformes).

\subsection{Observation du déroulement des ovulations}

\subsubsection{Préparation des géniteurs}

Entre deux saisons de ponte, les géniteurs sont regroupés dans un bassin en terre de $300 \mathrm{~m}^{2}$ alimenté par l'eau de la rivière Faye (environ 10 litres par seconde). Ils se nourrissent principalement d'aliment artificiel pour Saumon ( $49 \%$ de protéines), distribué en petits tas à des emplacements fixes. Compte tenu de la grande surface de bassin disponible (plus d'un mètre carré par poisson), ils ont à leur disposition une nourriture naturelle non négligeable (trichoptères à fourreaux décelés dans les estomacs).

Les poissons ont été pêchés après vidange du bassin le 18 mars 1983 et le 22 mars 1984, puis triés suivant le sexe : la couleur est plus sombre et la partie postérieure de la nageoire dorsale est plus développée chez les mâles, l'abdomen plus proéminent chez les femelles dont le coefficient de condition moyen (longueur à la fourche) était en 1983 de 1,21 contre 0,93 pour les mâles.

La fin de la maturation des géniteurs s'est déroulée dans des bacs carrés de $4 \mathrm{~m}^{2}$, en fibre de verre, à évacuation centrale et munis de couvercles semi-ouverts.

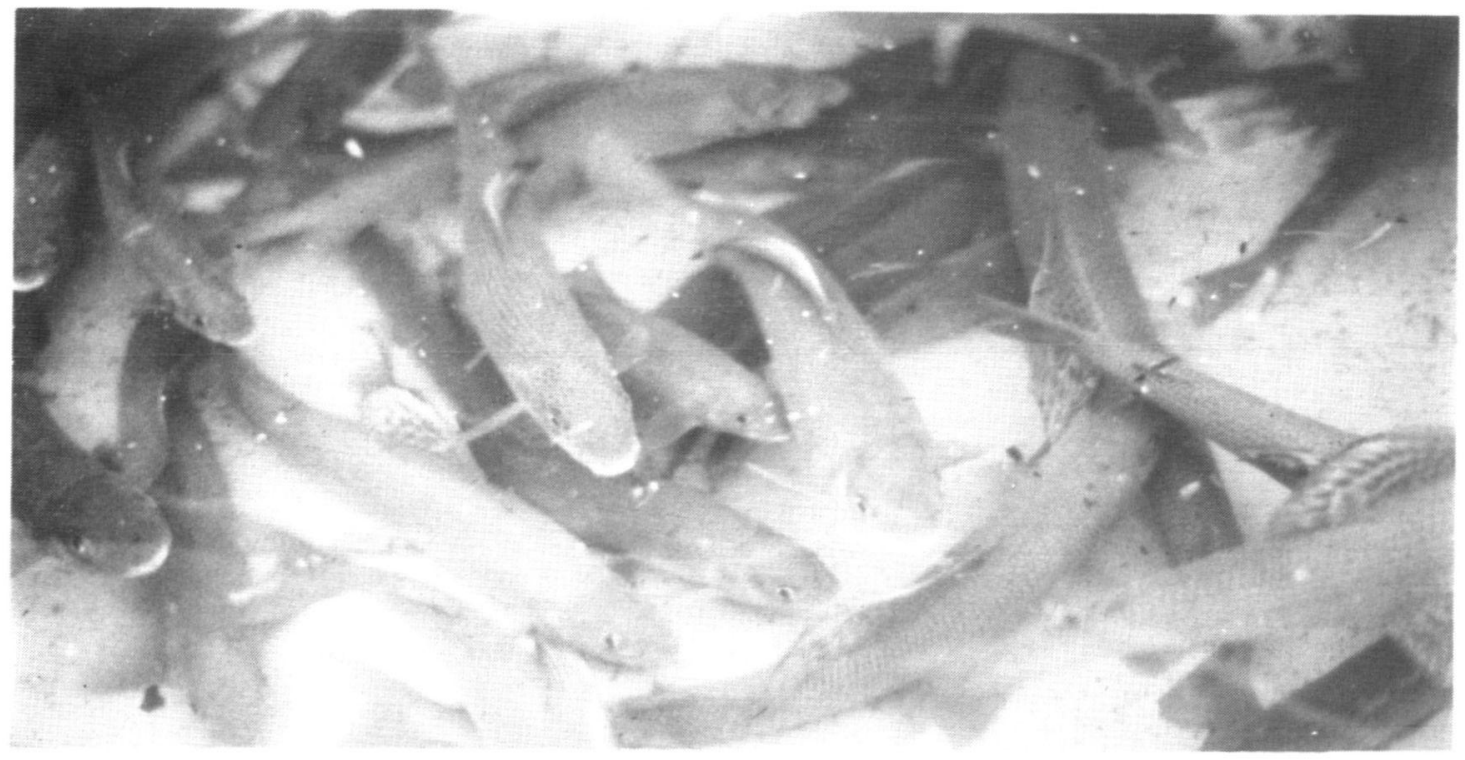

Photo 1 : Stockage des géniteurs avant la reproduction.

Photo 1 : Stabulation of spawners before the artificial spawning. 


\subsubsection{Contróles des ovulations}

L'état de maturité des femelles a été contrôlé après anesthésie rapide $(0,3 \mathrm{ml} / \mathrm{I}$ de Phénoxyéthanol) une fois par semaine en 1983. En 1984, la fréquence de contrôle a été de 5 jours jusqu'au 20 avril (température moyenne de l'eau $\bar{\theta}$, égale à $\left.6,5^{\circ} \mathrm{C}\right)$, puis de 3 jours $\left(\bar{\theta}=9,5^{\circ} \mathrm{C}\right.$ ).

Les femelles ovulées étaient placées dans un bassin d'attente du laboratoire jusqu'au moment de la reproduction artificielle, soit généralement dans l'heure suivante. Lorsque l'on ne pouvait prélever qu'une partie des ovules, l'autre partie étant encore rattachée sous forme d'une grappe décelable lors du massage de l'abdomen (femelle dite "partielle au premier passage"), on effectuait un deuxième prélèvement 3 jours après.

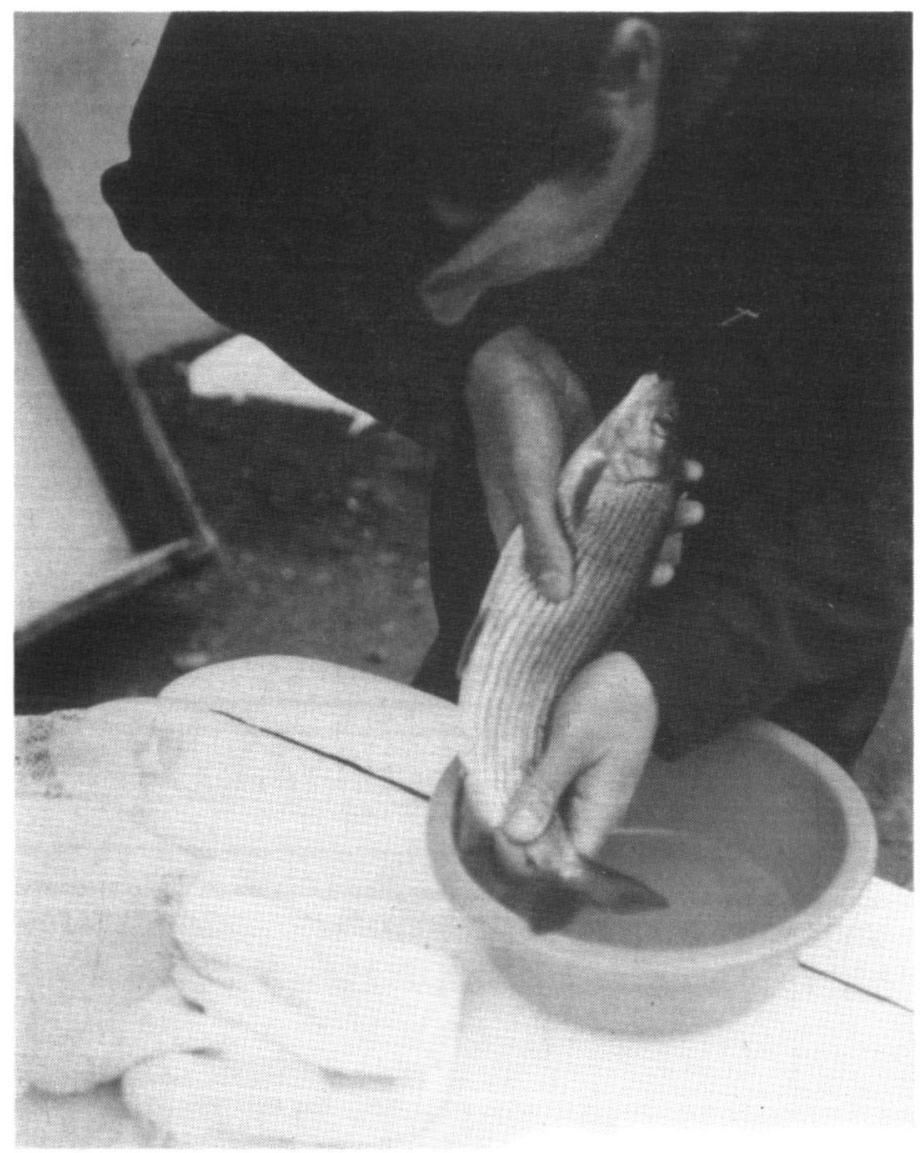

Photo 2 : Prélèvement des ovules.

Photo 2 : A female is stripped of its ova.

\section{3. Étude de la fécondité}

Nous avons calculé les moyennes des fécondités absolues (FA = nombre d'ovules par femelle), et des fécondités relatives ( $F R=F A / P$, avec $P=$ poids de la femelle avant ponte exprimé en $\mathrm{kg}$ ) pour 26 femelles de 4 ans et 68 femelles de 5 ans.

\subsection{Etude de la qualité des ovules}

La qualité des ovules a été appréciée à l'aide du pourcentage d'embryonnement des œufs à 150 degrés-jours, puis du pourcentage d'éclosion (*), calculés à partir d'échantillons de 200 ovules en 1983, et de pontes incubées séparément, ou regroupées par 2 ou 3, en 1984.

Chaque ponte a été fécondée avec un mélange de 4 ou 5 spermes à la dilution $10^{-2}$ dans du dilueur de sperme D.I.A. 532.

(*) Nombre d'alevins éclos divisé par le nombre d'œufs embryonnés. 


\subsection{Conditions de fécondation}

Une expérimentation a été réalisée en 1983 afin de vérifier l'intérêt de l'utilisation du dilueur de sperme D.I.A. 532.

Un mélange de 9 pontes a été divisé en deux lots de 8.000 ovules. Chaque lot a été fécondé par le même mélange de sperme à la dilution $10^{-2}$, l'un dans l'eau de la rivière, l'autre dans du dilueur préparé avec la même eau ; les pourcentages d'embryonnement et d'éclosion ont été étudiés.

\subsection{Conditions d'incubation}

A la pisciculture d'Augerolles les œufs d'O mbre sont incubés en clayette de type "californien". Ce système d'incubation ayant un rendement optimum quand les œufs sont disposés en monocouche. il nous a paru intéressant d'apprécier la charge optimale d'œufs par $\mathrm{m}^{2}$ de clayette. Pour cela nous avons étudié la cinétique du gonflement des œufs dans les heures suivant la fécondation.

\subsection{Essais de transposition de techniques connues chez la Truite}

\subsubsection{Conservation de sperme à court terme}

Dix mâles spermiants ont été isolés dans un bassin du laboratoire le 17 avril 1984 (Jo). A Jo + 1 nous avons prélevé $0,1 \mathrm{ml}$ de sperme par mâle, et le mélange a été conservé dans un sac plastique, sous atmosphère d'oxygène, à la température de la glace fondante, et à l'obscurité suivant la technique décrite par MAISSE et PORCHER en 1981 pour la Truite commune.

L'opération a été renouvelée à Jo +2 et Jo +3 avec les mêmes mâles.

A Jo $+3+6$ h, 4 lots de 500 à 600 ovules issus d'un mélange de plusieurs pontes ont été fécondés par un des trois mélanges de sperme, plus un fraîchement prélevé. Chacun des lots a été divisé en 2 ; les 8 lots obtenus ont été ensuite disposés au hasard dans une clayette à 8 compartiments. Les taux d'embryonnement et d'éclosion ont été étudiés.

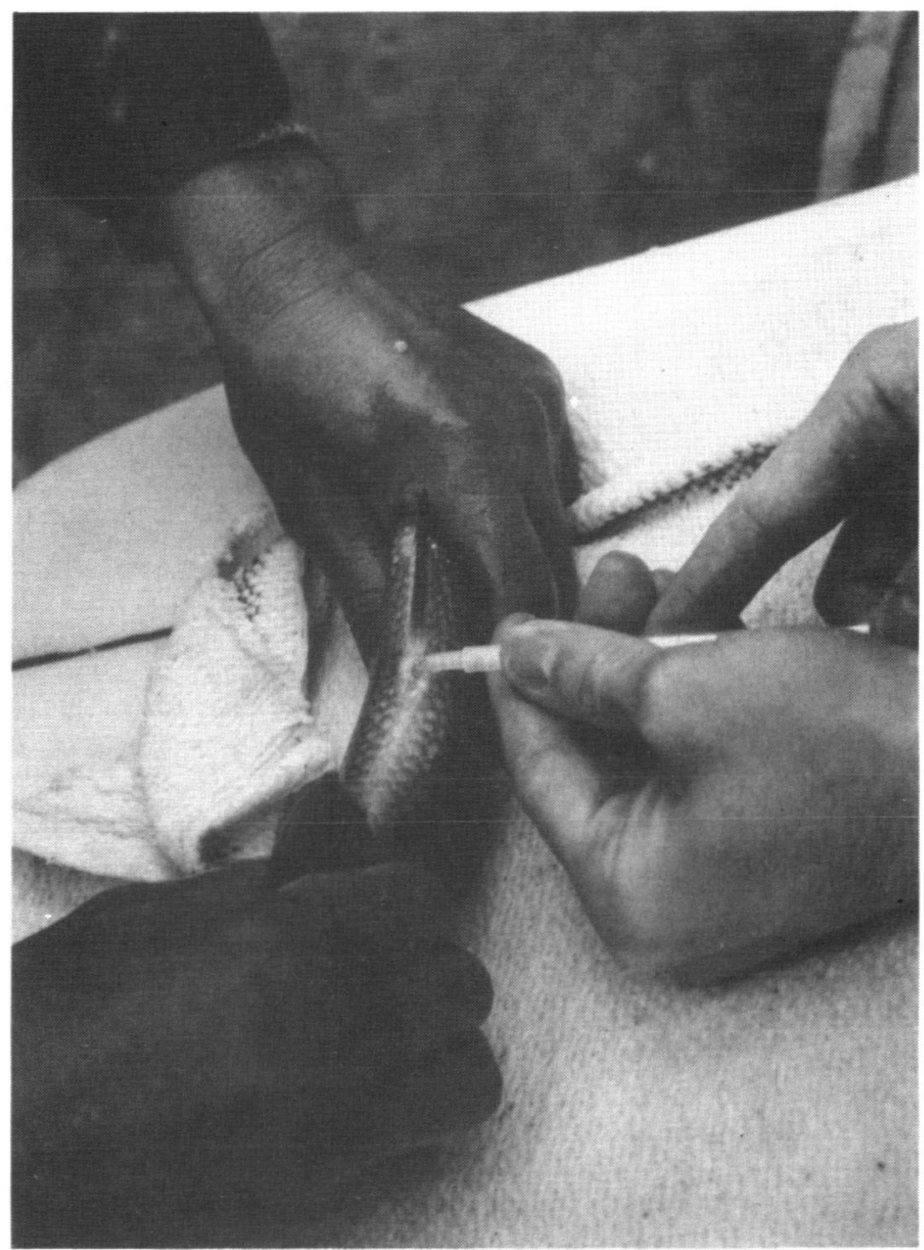

Photo 3 : Prélèvement du sperme au moyen d'une seringue Photo 3 : Punction of sperm with a squirt. 


\subsubsection{Fréquence optimale de contrôle de la maturité des femelles}

La détermination de la fréquence otpimale des contrôles de maturité des femelles nécessite l'étude du vieillissement des ovules laissés dans la cavité abdominale après l'ovulation suivant le protocole décrit par ESCAFFRE et BILLARD (1979) pour la truite arc-en-ciel. L'étude a porté sur 5 femelles de 4 ans choisies parmi un lot de femelles qui n'avaient pas encore ovulé 24 heures avant le début de l'expérience (premier prélèvement)

\subsubsection{Agitation des œufs dans les heures suivant la fécondation}

Les pontes de deux femelles de 4 ans ont été divisées en lots de 200 à 300 ovules, puis fécondées par un même mélange de sperme en présence de dilueur et transférées en eau douce où s'est opéré le durcissement de la coque.

Nous avons utilisé un agitateur linéaire animé de deux déplacements aller-retour de $3 \mathrm{~cm}$ par seconde, selon le protocole établi par BILLARD (1976), afin :

- de reproduire approximativement les conditions de transport : 2 heures après la fécondation, les différents échantillons ont été placés entre deux papiers humides dans des miniclayettes, et agités pendant 4, 10 ou 20 heures.

- de simuler les interventions du pisciculteur après le durcissement des cufs : à des temps variables après fécondation (entre 0.5 et 24 heures) un lot d'œufs de chaque femelle a été immergé dans l'eau d'une petite cuvette et agité pendant 10 minutes.

Les différents lots agités et les lots témoins ont été incubés séparément.

\section{3 - résultats}

\subsection{Observation du déroulement des ovulations (figures 1 et 2)}

En 1983, pendant la période d'ovulation (25 mars - 10 mai). la température de l'eau a varié de manière importante avec notamment deux chutes brutales à $3^{\circ} \mathrm{C}$ les 3 et 15 avril (figure 1).

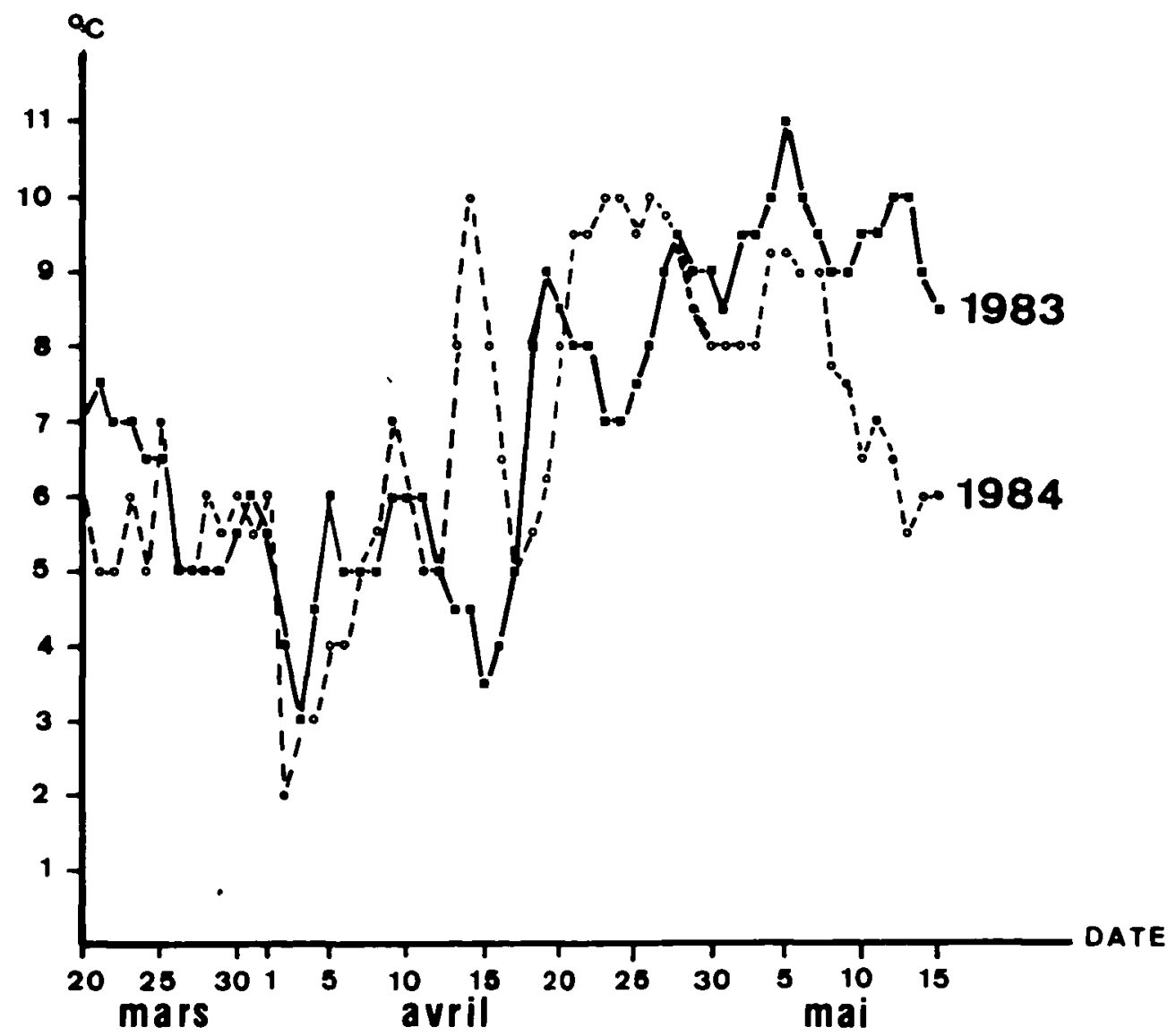

Figure 1 : Températures moyennes journalières de l'eau relevées entre le 20 mars et le 15 mai

Figure 1 : Daily mean temperatures of water noted between March the 20 th and May the 15 th. 
La courbe des fréquences cumulées des ovulations totales ou partielles au premier passage en fonction de la date (figure 2) montre un bon départ jusqu'au 1 er avril, puis un ralentissement dû au blocage de l'ovulation chez certaines femelles.

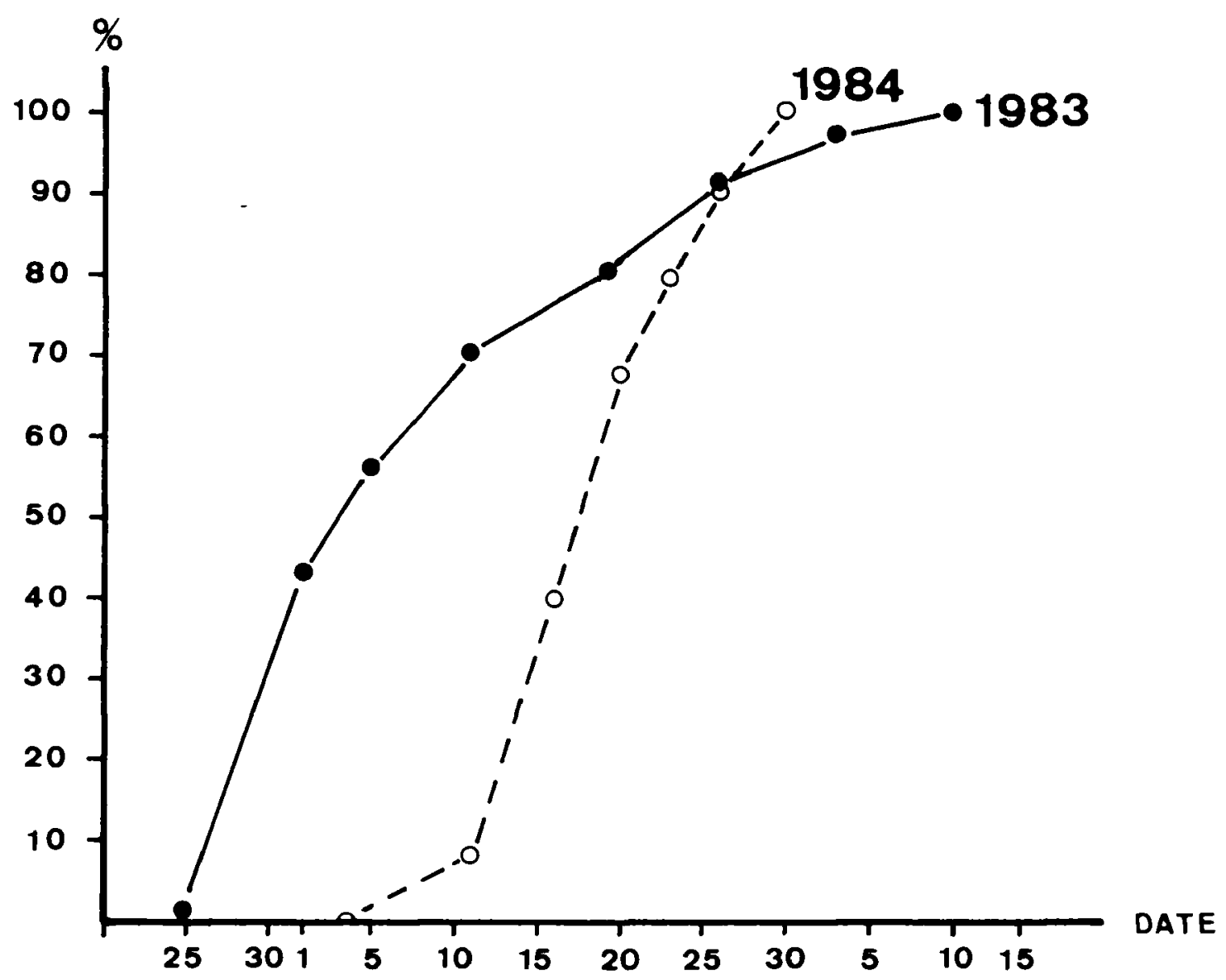

Figure 2 : Fréquences cumulées des ovulations en fonction de la date.

Figure 2 : Cumulated frequencies of ovulations according to the date.

L'autopsie des femelles n'ayant pas ovulé fait apparaître que les ovocytes ont maturé normalement, mais que l'ovulation ne s'est pas produite, la partie postérieure des ovaires étant par ailleurs très hémorragique.

En 1984, les ovulations ont commencé plus tard (11 avril) et pendant toute la période de ponte nous $n^{\prime}$ avons pas noté de baisse de la température en dessous de $5^{\circ} \mathrm{C}$. Les ovulations se sont étalées sur une vingtaine de jours, aucun ralentissement n'étant noté (figure 2).

\subsection{La fécondité}

Les moyennes des fécondités absolues et relatives sont respectivement de 3.623 ( $n=22$ $\sigma=1.412)$ et $10.509(n=22, \sigma=1.477)$ pour les femelles de 4 ans et de $3.755(n=45, \sigma=1.455)$ et 10.114 ( $n=45, \sigma=1.967)$ pour les femelles de 5 ans.

La fécondité absolue est correlée positivement au poids avant ponte $P(g)$ :

$$
F A=13 P-972 ; n=45, r=0,92
$$


3.3. Qualité des ovules (figure 3 , tableau 1)

\begin{tabular}{|c|c|c|c|c|c|}
\hline Annee & $\begin{array}{c}\text { caractéristique } \\
\text { de } \\
\text { l'ovulation }\end{array}$ & $n$ & $\begin{array}{c}\text { Pourcentage } \\
\text { moyen } \\
\text { d'embryonnement }\end{array}$ & $\begin{array}{l}\text { Pourcentage } \\
\text { moyen } \\
\text { d'éclosion }\end{array}$ & $\begin{array}{c}\text { Pourcent age } \\
\text { moyen de survie } \\
\text { de l'ovule } \\
\text { l'alevin } \\
\text { vésiculé }\end{array}$ \\
\hline \multirow{2}{*}{1983} & $\begin{array}{l}\text { Totale au } \\
\text { ler passage }\end{array}$ & 26 & 75 & 96 & 72 \\
\hline & $\begin{array}{l}\text { Partielle au } \\
\text { ler passage }\end{array}$ & 6 & 41 & 92 & 38 \\
\hline \multirow{3}{*}{1984} & $\begin{array}{l}\text { Totale au } \\
\text { ler passage }\end{array}$ & 45 & 85 & 95 & 81 \\
\hline & $\begin{array}{l}\text { Partielle au } \\
\text { ler passage }\end{array}$ & 11 & 92 & 95 & 87 \\
\hline & $\begin{array}{l}\text { Totale au } \\
\text { 2eme passage }\end{array}$ & 11 & 90 & 95 & 86 \\
\hline
\end{tabular}

Tableau 1 : Pourcentages moyens d'embryonnement, d'éclosion et de survie de l'ovule à l'alevin vésiculé pour les saisons de ponte 1983 et 1984 (échantillons de pontes incubés séparément en 1983, pontes incubées séparément ou regroupées par 2 ou 3 en 1984).

Table 1: Mean percentages of embryonic development, mean hatching rates and mean survival rates from ova to yolk sack stage noted in 1983 and 1984 (samples incubated separately ir. 1983, spawns incubated separately or grouped by 2 or 3 in 1984).
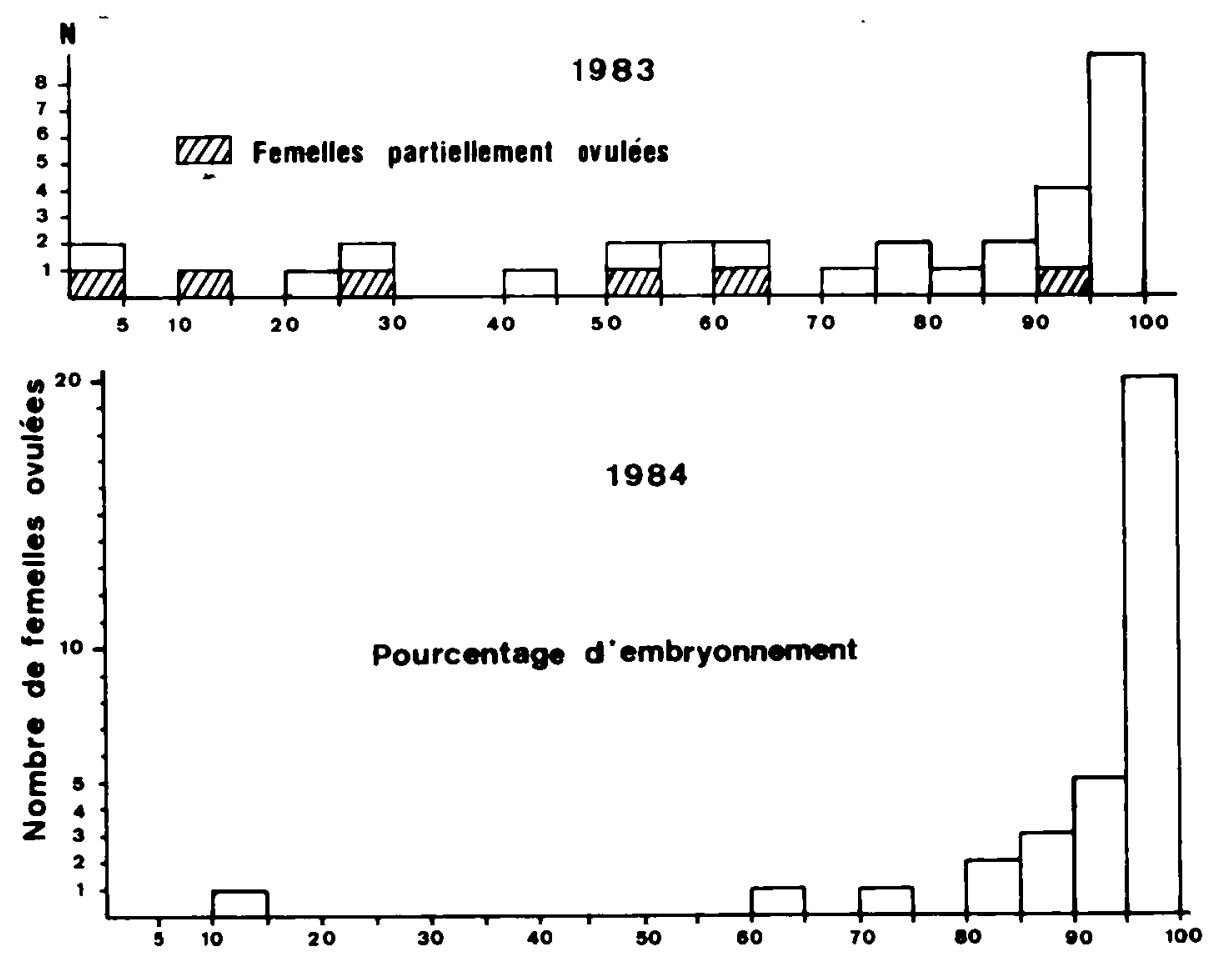

Figure 3: Histogramme des pourcentages d'embryonnement notés pour 33 femelles de 4 ans (1983) et 5 ans (1984).

Figure 3: Distribution of the percentages of embryonic development noted for 33 four years old and 33 five years old females. 
Le tableau 1 et la figure 3 montrent que la qualité des ovules est bonne en 1984, indépendamment de la caractéristique de l'ovulation (totale ou partielle). II existe cependant une variabilité importante au sein du lot de femelles en ce qui concerne la fécondabilité des ovules, appréciée avec le pourcentage d'embryonnement à 150 degrés-jours (figure 3 ).

Pour les femelles partielles au premier passage, il n'y a pas de différence significative entre les qualités des ovules du premier prélèvement et de la ponte "totale au deuxième prélèvement" effectuée 3 jours après (tableau 1 ).

Par ailleurs, aucune corrélation significative n'a été mise en évidence en 1983 entre les pourcentages d'embryonnement et d'éclosion et les caractéristiques morphologiques de la femelle (longueur, poids, coefficient de condition) ou le poids moyen des ovules.

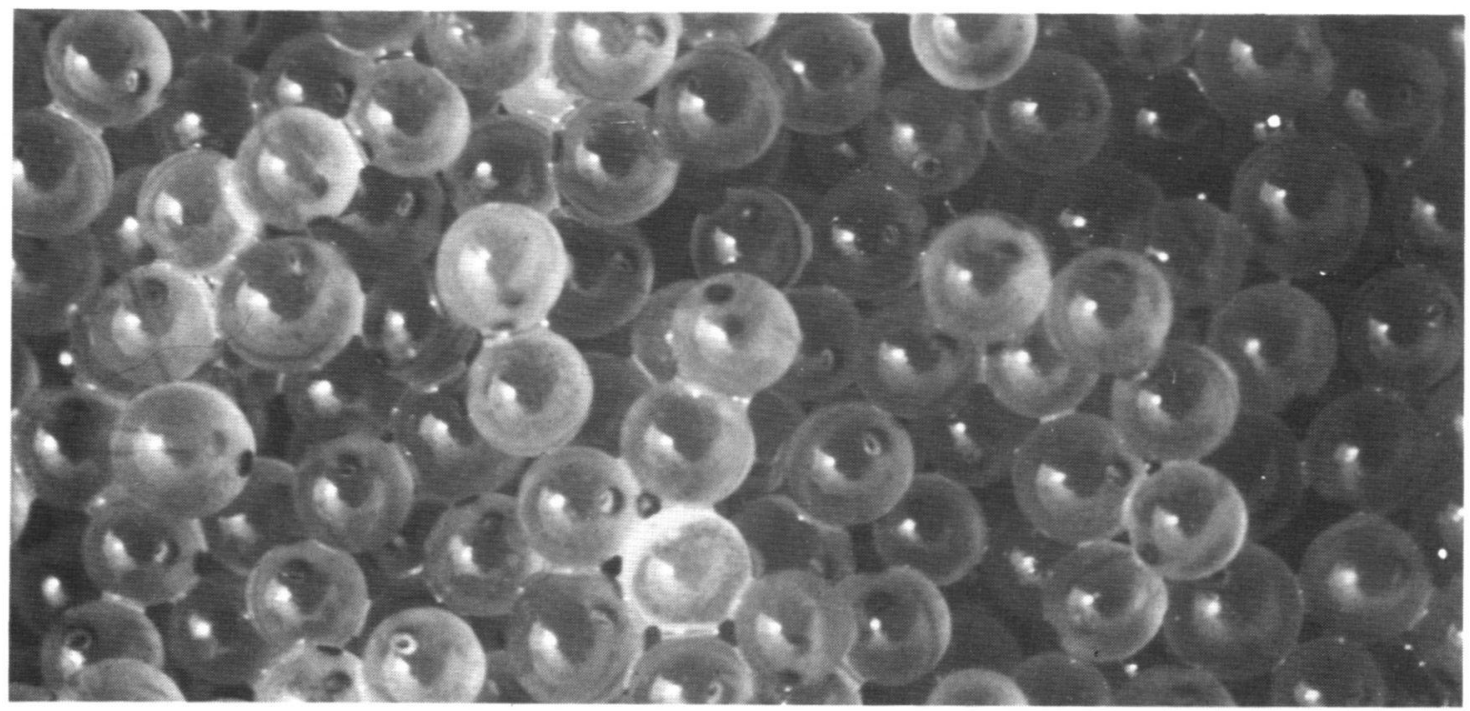

Photo 4 : Cufs embryonnés.

Photo 4 : Eyed eggs.

\subsection{Conditions de fécondation}

Le pourcentage d'embryonnement est meilleur avec le dilueur (92\%) qu'avec l'eau ( $83 \%$ ) ; le test du Khi deux est significatif $(\alpha=0,01)$.

Les pourcentages d'éclosion sont voisins : 99 et $98 \%$ respectivement.

\subsection{Conditions d'incubation}

3.5.1. Gonflement des œufs après fécondation (figure 4)

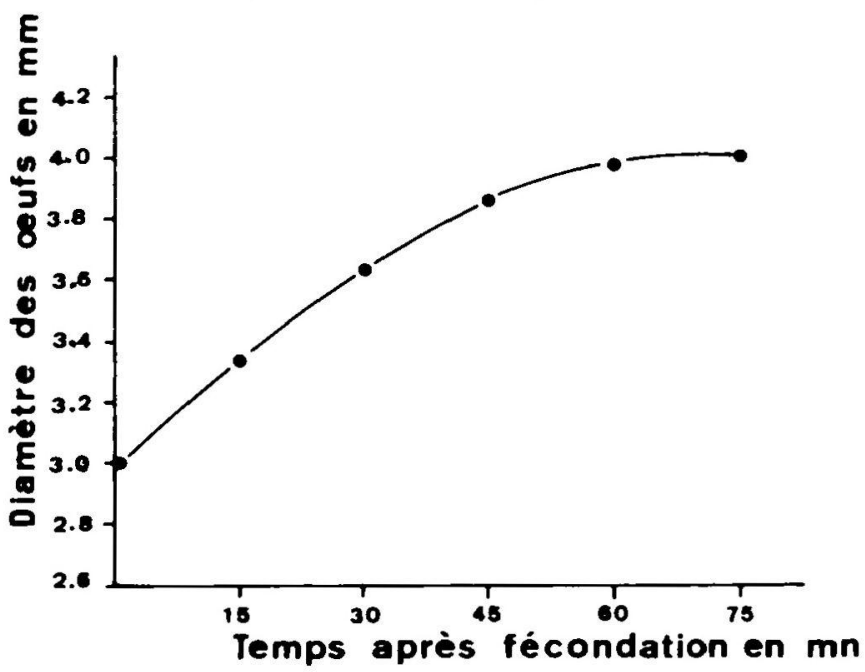

Figure 4: Cinétique du gonflement des œufs.

Figure 4 : Kinetics of the inflating of eggs. 
Après fécondation le diamètre de l'ceuf est voisin de $3 \mathrm{~mm}$. II se stabilise à $4 \mathrm{~mm}$ après 75 minutes de durcissement (figure 4) ; le volume a plus que doublé : il a varié de $0,014 \mathrm{~cm}^{3}$ à $0,034 \mathrm{~cm}^{3}$.

\subsubsection{Durée d'incubation}

En 1983 les premières éclosions ont eu lieu en moyenne 210 degrés-jours après la fécondation, les dernières intervenant au bout de 235 degrés-jours.

\subsection{Essais de transposition de techniques connues chez la truite}

3.6.1. Conservation du sperme à court terme (tableau 2)

\begin{tabular}{|c|c|c|c|c|c|}
\hline $\begin{array}{l}\text { Date de } \\
\text { prélève- } \\
\text { ment du } \\
\text { sperme }\end{array}$ & $\begin{array}{l}\text { Sous } \\
\text { Lot }\end{array}$ & $\begin{array}{c}\text { Nombre } \\
\text { d'ovules }\end{array}$ & $\begin{array}{l}\text { Pourcent age } \\
\text { d'embryonnement }\end{array}$ & $\begin{array}{l}\text { Pourcentage } \\
\text { d'eclosion }\end{array}$ & $\begin{array}{l}\text { Pourcentage } \\
\text { de survie de } \\
\text { l'ovule } \\
\text { a l'alevin } \\
\text { vésiculé }\end{array}$ \\
\hline \multirow{2}{*}{ Jo +1} & I & 270 & 97,7 & 96,9 & 94,8 \\
\hline & II & 312 & 96,8 & 98 & 94,8 \\
\hline \multirow{2}{*}{$30+2$} & III & 266 & 97,7 & 98,8 & 96,6 \\
\hline & IV & 296 & 98,3 & 97,2 & 95,6 \\
\hline \multirow{2}{*}{$\mathrm{Jo}+3$} & v & 258 & 95,3 & 98,3 & 93,8 \\
\hline & VI & 275 & 97,8 & 98,5 & 96,3 \\
\hline \multirow{2}{*}{$\begin{array}{l}\text { Jo+3+6 h } \\
\text { (témoin) }\end{array}$} & VII & 245 & 95,9 & 99,1 & 95,1 \\
\hline & VIII & 296 & 95,6 & 97,8 & 93,5 \\
\hline
\end{tabular}

Tableau 2 : Conservation du sperme à court terme : pourcentage d'embryonnement et d'éclosion notés pour les 8 sous lots.

Table 2 : : Preservation of sperm during a short time : percentages of embryonic development and hatching rates noted for the 8 samples.

Les pourcentages d'embryonnement et d'éclosion des 6 sous lots avec du sperme conservé de 6 à 54 heures à $2^{\circ} \mathrm{C}$ sont supérieurs à $95 \%$ (tableau 2 ) et ne diffèrent pas de façon significative des deux sous lots témoins.

\subsubsection{Vieillissement des ovules in vivo (figure 5)} (figure 5).

Les pourcentages d'embryonnement et d'éclosion chutent 7 jours après le début de l'ovulation

Pour la femelle B on note un faible pourcentage d'embryonnement lors du premier prélèvement qui s'est situé au tout début de l'ovulation. Les ovules de la femelle $E$ vieillissent plus rapidement en raison de la qualité médiocre de la ponte : le pourcentage d'embryonnement chute de 80 à $0 \%$ en une semaine. 

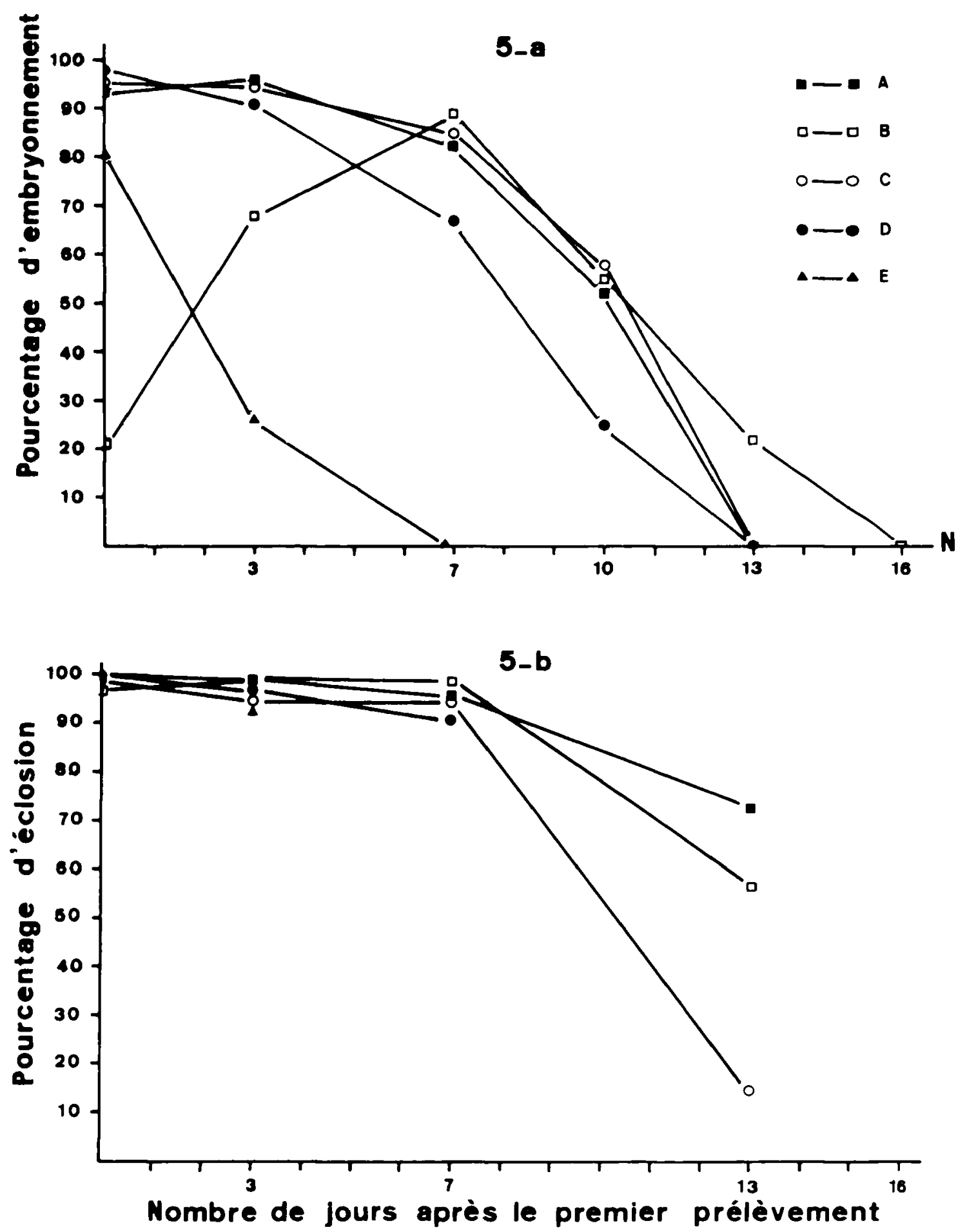

Figure 5 : Pourcentages d'embryonnement (5-a) et d'éclosion (5-b) en fonction de la durée de séjour post ovulatoire des ovules dans la cavité abdominale (5 femelles). Le premier prélévement se situe 0 à 24 h après le début de l'ovulation.

Figure 5: Percentages of embryonic development (5-a) and hatching rates (5-b) according to the duration of stay of eggs in the abdominal cavity in the post ovulatory period. First sampling occurs in the first 24 hours after the beginning of the ovulation. 


\subsubsection{Agitation des ceufs dans les heures suivant la fécondation (figures 6 et 7)}

La mauvaise qualité de la ponte d'une femelle (pourcentage d'embryonnement du lot témoin inférieur à $10 \%$ ) nous amène à analyser avec prudence les résultats obtenus pour la seconde femelle. Ceux-ci semblent indiquer que, quel que soit le type d'agitation, les pourcentages d'embryonnement et d'éclosion des lots agités. supérieurs respectivement à 90 et $95 \%$, ne diffèrent pas sensiblement de ceux des lots témoins non agités (figures 6 et 7).

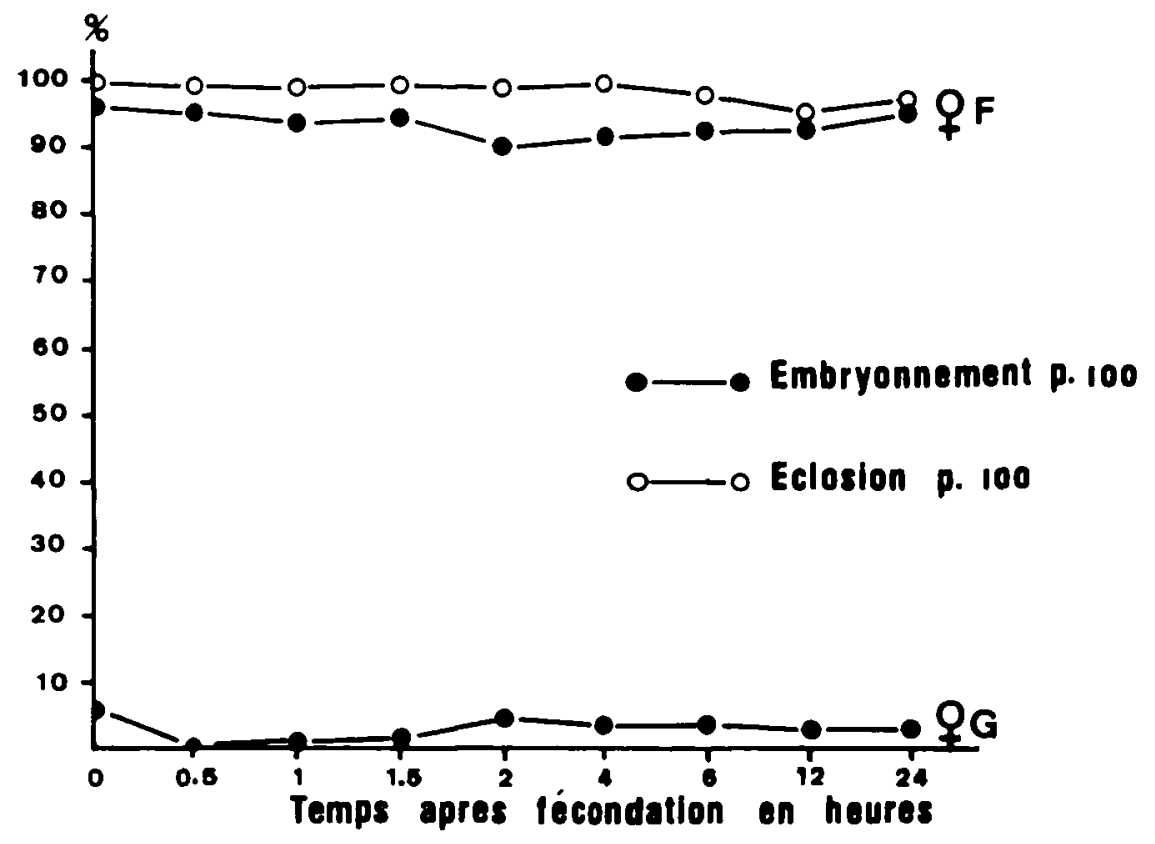

Figure 6 : Effet de l'agitation humide sur les pourcentages d'embryonnement et d'éclosion.

Figure 6 : Influence of the agitation in water on the percentage of embryonic development and the hatching rate.

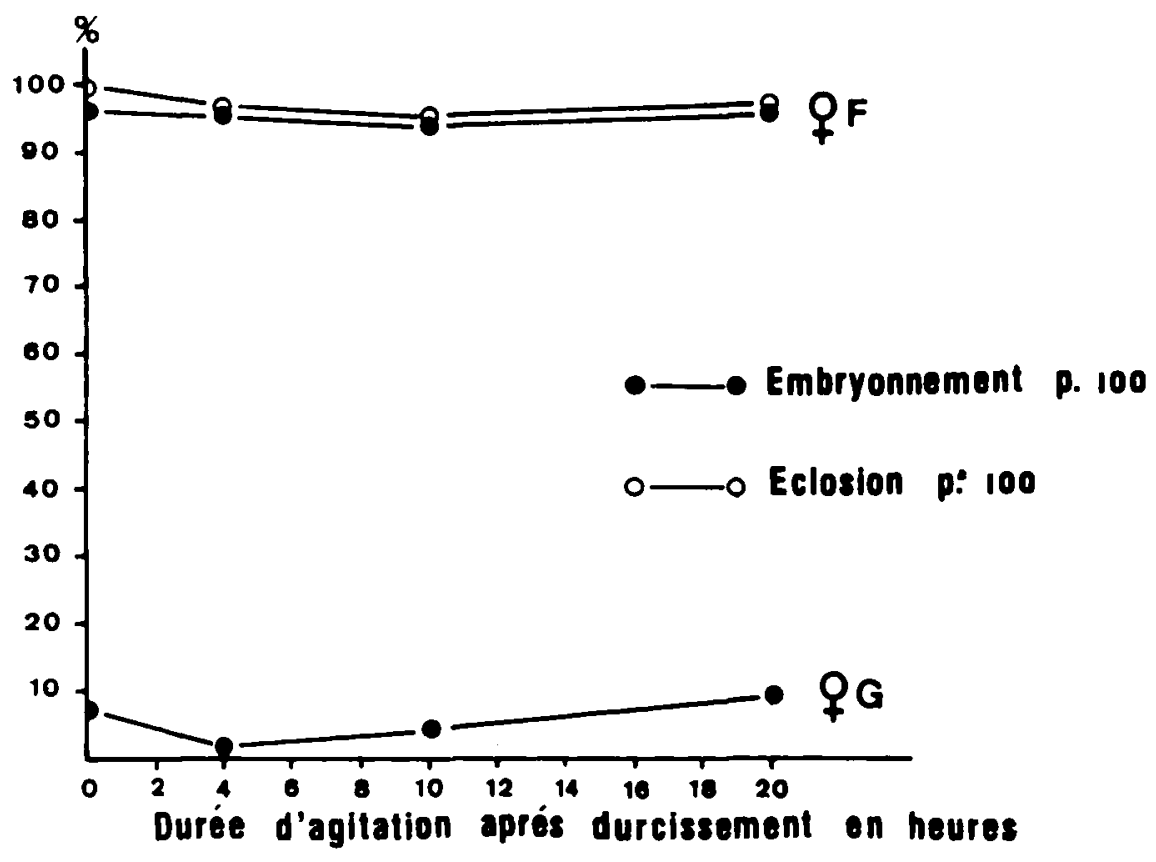

Figure 7: Effet de l'agitation à sec sur les pourcentages d'embryonnement et d'éclosion.

Figure 7 : Influence of the agitation out of water on the percentage of embryonic development and the hatching rate. 


\section{$4 \cdot$ DISCUSSION}

\subsection{Les géniteurs et le contrôle des ovulations}

Les sujets élevés en milieu artificiel ont une vie sexuelle semblable à celle des individus sauvages. Ainsi les géniteurs de troisième génération en pisciculture ont donné des produits sexuels à l'âge de 3 ans, certains étant mâtures à 2 ans, et ce malgré une croissance plus faible que dans les rivières d'Auvergne (*) en raison de la température de l'eau (inférieure à $5^{\circ} \mathrm{C}$ pendant 5 à 6 mois). PERSAT (1978) a noté sur 76 individus de deux étés capturés entre 1976 et 1978 dans l'Ain (Franche Comté) la même proportion de sujets matures chez les mâles que chez les femelles, soit $35 \%$; le rapport était de $100 \%$ dans les deux sexes pour 20 sujets de trois étés ou plus.

La période de ponte est liée à la température de l'eau. De 1982 à 1984 les premières ovulations ont eu lieu de 127,5 à 134 degrés-jours après la date du 1 er mars (tableau 3 ).

\begin{tabular}{|c|c|c|c|c|c|c|}
\hline & \multicolumn{2}{|c|}{1982} & \multicolumn{2}{|c|}{1983} & \multicolumn{2}{|c|}{1934} \\
\hline $\begin{array}{l}\text { Période I : du } \\
\text { ler mars jusqu'a } \\
\text { la premiere } \\
\text { ovulation. } \\
\text { Période II : du } \\
\text { ler mars jusqu'a } \\
\text { la moitié des } \\
\text { ovulations }\end{array}$ & I & II & I & II & I & I I \\
\hline $\begin{array}{l}\text { Températures } \\
\text { moyennes journe- } \\
\text { lières extrêmes } \\
\left({ }^{\circ} \mathrm{C}\right)\end{array}$ & $2-5,5$ & $2-8$ & $3-7,5$ & $3-7,5$ & $0,1-7$ & $0,1-10$ \\
\hline $\begin{array}{l}\text { Moyenne des } \\
\text { temperratures } \\
\text { journalieres } \\
\left({ }^{\circ} \mathrm{C}\right)\end{array}$ & 4 & 4,5 & 5,6 & 5,4 & 3,8 & 4,3 \\
\hline $\begin{array}{l}\text { Nombre de } \\
\text { degrés-jours }\end{array}$ & 127,5 & 179 & 134 & 184 & 131 & 187 \\
\hline
\end{tabular}

Tableau 3: Relation entre le déroulement des ovulations et la température de l'eau depuis le 1 er mars. En 1982 les géniteurs n'étaient pas les mêmes qu'en 1983 et 1984 . En 1984, les dates correspondant à la 1 ère ovulation et à $50 \%$ des ovulations ont été estimées d'après la courbe des fréquences cumulées des ovulations.

Table 3 : Relation between occurrence of ovulations and water temperature from March the 1 st. In 1982, spawners were not the same as in 1983 and 1984 . In 1984, the dates fitting with the first ovulation and $50 \%$ of ovulations have been estimated from the curve of cumulated frequencies of the ovulations.

Il semblerait d'après l'analyse des températures de l'eau et de la chronologie des ovulations en 1983 et 1984 qu'une température dépassant 4,5 à $5^{\circ} \mathrm{C}$ en fin de période de maturation (fin mars, avril) soit un des facteurs nécessaires au déclenchement de l'ovulation ; cela confirmerait les indications de D'HULSTERE et PHILIPPART (1982), selon lesquelles la reproduction de l'Ombre a lieu en mars et avril lorsque la température de l'eau passe de $4-5^{\circ} \mathrm{C}$ à $8-9^{\circ} \mathrm{C}$. Une chute brutale de la température de l'eau en-dessous de $5^{\circ} \mathrm{C}$ après le 15 mars s'accompagne d'un ralentissement de la maturation et du blocage des ovulations chez certaines femelles.

Les pontes de 1985 devraient permettre d'affiner ces observations.

Des essais de synchronisation de pontes par traitements hormonaux rapportés par différents auteurs ont donné des résultats divergents. Ainsi des injections d'hormones synthétiques (D'HULSTERE et PHILIPPART, 1982) et d'extraits hypophysaires de carpe (BILLARD, 1981) sur des ombres sauvages capturés au moment du frai se sont soldées par un échec. STEIN et WINTERSPERGER (1980) ont par contre réussi, avec des injections similaires, à réduire sensiblement la période d'ovulation des lots traités par rapport au lot témoin.

(*) Les individus mesurent $33 \mathrm{~cm}$ en moyenne à 3 ans sur l'Allagnon et l'Allier. 


\subsection{Les gametes}

Le nombre d'ovules par kilogramme de poids vif après ponte, 10.100 pour les femelles de 5 ans, est beaucoup plus élevé que chez les autres salmonidés $\left(^{*}\right)$ et diffère sensiblement du chiffre avancé par SVETINA (1958) : 6 a 7.000 par kilogramme de poids vif chez les individus sauvages. Le diamètre des ovules est par contre plus faible que chez la truite commune : $3 \mathrm{~mm}$ en moyenne contre 4 à $5 \mathrm{~mm}$.

Après l'ovulation, la qualité des ovules laissés dans la cavité générale se conserve pendant 3 à 7 jours $\left(3^{\circ} \mathrm{C}<\bar{\theta}<7^{\circ} \mathrm{C}\right.$ ) ce qui est une durée intermédiaire entre celle obtenue pour le brochet, 2 jours à $12^{\circ} \mathrm{C}$ (BRY et al., 1978) et celle observée chez la truite arc-en-ciel, 8 à 15 jours avec $6.5^{\circ} \mathrm{C}<\overline{0}<13^{\circ} \mathrm{C}$ (ESCAFFRE et BILLARD, 1979).

Les mâles donnent une faible quantité de laitance et il est préférable de la prélever au moyen d'une seringue. Comme pour la truite le sperme peut être conservé au moins 72 heures à $2^{\circ} \mathrm{C}$ sous atmosphère d'oxygène sans altération de sa qualité, alors que les tentatives de congélation n'ont pas été fructueuses (STEIN et WINSTERSPERGER, 1980).

Enfin les essais comparatifs de fécondation dans l'eau et dans le dilueur d'insémination confirment l'effet bénéfique du dilueur noté chez la truite par PETIT et al. (1973). II n'a pas été fait de comparaison avec la fécondation sans eau, dans le liquide cœlomique.

\subsection{L'incubation}

Les résultats des expérimentations sur l'agitation des œufs à sec et en atmosphère humide vont dans le sens des observations faites sur la truite arc-en-ciel par BILLARD (1976 a et b), qui ne note pas de diminution significative du taux d'embryonnement lorsque les œufs sont agités entre la première et la vingtième heure après l'insémination.

L'incubation a nécessité $2 \underline{00}$ degrés-jours en 1982 (dont 110 jusqu'à l'apparition des yeux) pour une température moyenne $(\bar{\theta})$ de $8,5^{\circ} \mathrm{C}$. En 1983, elle en a nécessité 220 (190 à 230 selon les pontes) $\left(\bar{\Theta}=9^{\circ} \mathrm{C}\right.$. Ces valeurs se situent plutôt au-dessus de celles citées dans la littérature

- 200 degrés-jours (BQUSCHKIEL, in VIVIER, 1958)

- 180 degrés-jours $\left(\bar{\theta}=7^{\circ} \mathrm{C}\right)$ (VIVIER, 1958)

- 177 degrés-jours $\left(\bar{\theta}=8^{\circ} \mathrm{C}\right)$ (D'HULSTERE et PHILIPPART, 1982).

JUNGWIRTH et WINKLER (1984) indiquent la durée d'incubation (y en jours) en fonction de la température $\left(x\right.$ en $\left.^{\circ} \mathrm{C}\right)$ :

$$
\dot{y}=6484.6 /(x+5.103)^{2.099}
$$

Pour des températures de 8.5 et $9^{\circ} \mathrm{C}$ on obtient avec cette formule des nombres de degrés-jours respectivement égaux à 230 et 225 .

Le pourcentage de survie de l'ovule à l'alevin vésiculé observé en 1984 pour les pontes de 65 femelles de 5 ans (239.000 ovules) est de $80,8 \%$. Ce taux résulte d'un progrès au cours de ces dernières années $(57,6 \%$ en 1981), mais reste inférieur aux valeurs observées à Augerolles pour le Saumon atlantique (géniteurs sauvages), soit 85,7 et 87,1\% pour les contingents 1982 et 1983

La durée moyenne de résorption est de 90 degrés-jours; 40 à 50 degrés-jours après l'éclosion, à une taille moyenne de $15 \mathrm{~mm}$, les alevins commencent à gober les particules

\section{5 - CONCLUSIONS - APPLICATIONS PRATIQUES}

Les expériences réalisées en 1982 et 1983 ont fourni divers résultats d'ordre physiologique présentés et discutés aux chapitres 3 et 4 . Jointes aux observations et essais plus empiriques réalisés à la Salmoniculture d'Augerolles depuis une dizaine d'années, elles montrent qu'il est tout à fait possible de maîtriser en captivité le cycle reproducteur de l'Ombre commun.

Nous résumons ci-après les techniques que nous utilisons maintenant dans cette pisciculture pour l'élevage des géniteurs et la pratique de la reproduction artificielle. Notons toutefois que ces techniques seront à perfectionner ou affiner en fonction d'expériences à poursuivre, et qu'elles nécessitent probablement d'être plus ou moins adaptées aux conditions d'autres salmonicultures.

\subsection{Choix et entretien des géniteurs}

- Les futurs géniteurs sont choisis par sélection massale parmi la descendance de sujets sauvages de souche locale capturés en période de frai.

- Les reproducteurs sont utilisés pour la première fois à l'âge de 3 ans.

(*) 1.800 pour les saumons de l'Allier et 3.050 pour les truites communes élevées à Augerolles. 
- La gestion du cheptel est facilitée par un marquage distinctif de chaque cohorte (bleu alcyan sur les nageoires) avant introduction dans le bassin à reproducteurs.

- Entre deux saisons de ponte, l'intervention du pisciculteur se limite à la distribution d'aliment artificiel, ce qui diminue les risques de stress. On complémente la ration avec un mélange vitaminique pour géniteurs, 3 jours par mois.

\subsection{Manipulation des géniteurs}

- Les géniteurs sont transférés dans des bacs à parois lisses quelques jours avant la date présumée des premières ovulations, qui interviennent pour un nombre de degrés-jours après le 1 er mars proche de 130.

- Ils sont anesthésiés $(0,3 \mathrm{ml} / /$ de Phénoxyéthanol) lors des contrôles de la maturité : la fréquence des manipulations est liée à la température de l'eau :

- tous les 3 jours avec $\bar{\theta}>7,5^{\circ} \mathrm{C}$;

- tous les 5 jours avec $5^{\circ} \mathrm{C} \leqslant \bar{\theta} \leqslant 7^{\circ} \mathrm{C}$; en cas de chute brutale de la température on ne compte pas les jours où elle est inférieure à $4,5^{\circ} \mathrm{C}$.

- Les géniteurs sont épongés délicatement afin d'éviter le contact de l'eau avec les gamètes.

- Les pontes sont recueillies et si possible incubées séparément, afin de ne pas mélanger celles de qualité médiocre à celles de bonne qualité, donc de réduire ensuite le travail de tri des œu fs morts.

- Lorsqu' une partie des œufs est encore attachée en grappe (ponte partielle), on ne doit pas forcer la femelle, mais effectuer un deuxième prélèvement 3 jours après. Les derniers ovules sont évacués 3 jours après la ponte "totale".

\subsection{Fécondation}

- La méthode de fécondation artificielle est semblable à celle appliquée à la truite.

- La laitance est prélevée au moyen d'une seringue. On peut la conserver dans un récipient sec, sous atmosphère d'oxygène, à la température de la glace fondante.

- En présence de dilueur 532, on féconde $100 \mathrm{ml}$ d'ovules avec $1 \mathrm{ml}$ de sperme.

- Cinq minutes après la fécondation, on rince les œufs pour éliminer les excédents de sperme et le sang

\subsection{Incubation}

- Le durcissement s'opère dans des clayettes de type californien (perforations de $1,5 \mathrm{~mm}$ ), où la mise en charge ne doit pas dépasser 400 œufs au $\mathrm{dm}^{2}\left(40.000\right.$ au $\left.\mathrm{m}^{2}\right)$ afin d'éviter leur superposition.

- Les œufs morts sont retirés dans les 24 heures suivant la fécondation, puis quotidiennement après l'apparition des yeux. Cette mesure sanitaire est associée à des bains de vert de malachite (5 $\mathrm{mg} / \mathrm{l}$, une fois tous les deux jours).

Depuis 1978, la plus grande partie des jeunes ombres produits à la Salmoniculture d'Augerolles est libérée dans divers cours d'eau de l'Auvergne et du Limousin, juste après résorption de la vésicule. ou au stade "ombrette", après mise en grossissement pendant quelques mois. Les modes d'élevage utilisés à Augerolles, décrits sommairement par CARMIE et CUINAT (1984), feront l'objet d'une prochaine mises au point.

\section{Remerciements}

Monsieur JALABERT, Maître de Recherche à I'I.N.R.A., a fourni des conseils utiles lors des premières pontes artificielles à Augerolles.

Messieurs LOMBARDY et MESTAS, employés à la Salmoniculture, ont participé aux activités d'élevage et d'expérimentation. Ce dernier employé est mis à la disposition du C.S.P. par les sept Fédérations Départementales d'A.A.P.P. de l'Auvergne et du Limousin, en vue de contribuer au développement de l'élevage de l'Ombre commun pour le repeuplement de certains cours d'eau de cette région

\section{BIBLIOGRAPHIE}

BILLARD R., 1976a. Effet de l'agitation des ovules et des œufs de Truite arc-en-ciel sur la fécondabilité et le début du développement embryonnaire. Bull. Fr. Pisc., 262, 5-11. 
BILLARD R., 1976b. Sensibilité des œufs de Truite arc-en-ciel aux chocs mécaniques pendant la phase de durcissement. Bull. Fr. Pisc., 263, 45-49.

BILLARD R., 1981. La pisciculture d'étang en Bavière. Pisc. Fr., 65, 13-24.

BRY C., BILLARD R., de MONTALEMBERT G., 1978. Induction de la maturation ovocytaire et de l'ovulation par traitement hormonal chez le Brochet (Esox Lucius) Bull. Fr. Pisc., 271, 21 -32

CARMIE H., CUINATR., 1984. Élevage de l'Ombre commun. Premiers résultats obtenus à la Salmoniculture du Conseil Supérieur de la Pêche à Augerolles. Dactylographié, 19 p.

CUINAT R., 1978. La salmoniculture du Conseil Supérieur de la Pêche à Augerolles. Dactylographié, $15 \mathrm{p}$.

D'HULSTERE D., PHILIPPART J.C., 1982. Observations sur le comportement d'éclosion et de postéclosion chez l'O mbre commun, Thymallus thymallus (L.). Cahiers d'Ethologie Appliquée 2, (1). 63-80.

ESCAFFRE A.M., BILLARD R., 1979. Evolution de la fécondabilité des ovules de Truite arc-en-ciel (Salmo gairdneri) laissés dans la cavité abdominale au cours de la période post ovulatoire. Bull. Fr. Pisc., 272, 56-70.

HUET M., 1949. Apercu des relations entre la pente et les populations piscicoles des eaux courantes. Schweiz. Z. Hydrol., XI, 332-351.

JUNGWIRTH M., WINKLER H., 1984. The temperature dependance of embryonic development of Grayling(Thymallus thymallus), Danube salmon(Hucho hucho), Arctic char(Salvelinus alpinus) and Brown trout (Salmo trutta fario). Aquaculture, 38, 315-327.

MAISSE G., PORCHER J.P., 1981. Une méthode de production de truites fario destinées au repeuplement de restauration. Bull. Fr. Pisc., 280, 134-144.

PERSAT H., 1976. Principaux aspects de l'écologie de l'Ombre commun. Thèse de Doctorat de 3ème cycle de Sciences Biologiques, Université Claude Bernard Lyon I, $69 \mathrm{p}$.

PERSAT H., 1978. Ecologie de l'Ombre commun. II. Compte rendu des travaux 1977-1978. Ecole Nationale Vétérinaire de Lyon et Université Claude Bernard Lyon I, $40 \mathrm{p}$

PETIT J., JALABERT B., CHEVASSUS B., BILLARD R., 1973. L'insémination artificielle de la Truite (Salmo gairdneri Richardson). I. Effet du taux de dilution, du pH et de la pression osmotique du dilueur sur la fécondation. Ann. Hydrobiol., 4, 201-210.

PHILIPPART J.C., D'HULSTERE D., 1976. La pisciculture de l'Ombre. Gazette Officielle de la Pêche, 563, 13-14

STEIN H., WINTERSPERGER R., 1980. The artificial insemination of the grayling/Thymal/us thymallus L.). 9th International Congress on Animal Reproduction, Madrid, vol. II, 311-315.

SVETINA M., 1958. L'Ombre commun et sa reproduction artificielle. Bull. Fr. Pisc., 191, 60-65.

VIVIER P., 1958. L'Ombre commun (Thymallus thymallus L.) sa reproduction et son élevage. Bull. Fr. Pisc., 191, 45-58 